bleibenden restlichen Stämme wuchsen zwar auch ohne die betreffenden Substanzen mehr oder weniger stark, jedoch war ihre Anwesenheit zu optimalem Wachstum notwendig.

Schließlich ist in den Abb. 1 und 2 die Wirkung von Lactoflavin bzw. Pantothensäure in Abhängigkeit von ihrer Konzentration auf das Wachstum von 2 Stämmen (IGAu und $1521 \mathrm{Au}$ ) dargestellt, die beide Lactoflavin und Pantothensäure unbedingt benötigen. Die Lactoflavinkurven (Abb. 1) sind recht steil, ihre Leerwerte sehr niedrig; halboptimales Wachstum wurde mit etwa 0,3·10-7 $\mathrm{g}$ Lactoflavin $/ \mathrm{cm}^{3}$ erreicht; die Stämme dürften sich also für Lactoflavinteste durchaus eignen.

Weniger geeignet erscheinen die beiden Stämme für die Bestimmung von Pantothensäure. Die Leerwerte sind recht hoch, was aber wohl auf einem geringen Gehalt des Nährmediums an Pantothensäure beruht, da sie bei allen Stämmen in dieser Höhe lagen. Vor allem aber ist die Steilheit der Kurven gering, die sich in ihrem linearen Teil über ein Konzentrationsgebiet von 21 bis $330 \mathrm{~m} \gamma$ d, l-Ca-Pantothenat $/ \mathrm{cm}^{3}$ erstrecken. Halboptimales
Wachstum wurde bei einer Konzentration an $d, l$ Ca-Pantothenat von etwa $0,7 \cdot 10^{-7} \mathrm{~g} / \mathrm{cm}^{3}$, optimales erst bei $3,3 \cdot 10^{-7} \mathrm{~g} / \mathrm{cm}^{3}$ gefunden. Verglichen mit der bei Streptobacterium plantarum $10 \mathrm{~S}$ optimal wirkenden Konzentration von $2 \cdot 10^{-8} \mathrm{~g}$ (+) Pantothensäure $/ \mathrm{cm}^{327}$, bei etwa gleichem oder sogar stärkerem Wachstum, deuten die für Staphylokokken gefundenen, weit höheren Konzentrationen darauf hin, daß dem Nährmedium noch Substanzen fehlen, die etwa für die Ausnützung der Pantothensäure von Bedeutung sein mögen.

Für technische Mitarbeit danken wir Frl. E. Ma n n s.

Während der Korrektur ist uns noch eine Arbeit von Evans ${ }^{28}$ über die Ernährung von Staphylokokken bekannt geworden. Von 40 untersuchten Stämmen benötigten 39 Aneurin, 38 Nicotinsäure, 21 Biotin, 2 Adermin, 7 Pantothensäure, 0 Lactoflavin, 0 Folinsäure.

Bei einigen in der letzten Zeit von uns untersuchten Staphylokokkenstämmen erwies es sich als notwendig, Citrat durch Acetat zu ersetzen, da Citrat zu stark, oft total hemmte.

27 E. F. Möll e r, zitiert bei R. Kuhn u. Th. Wi e 1 and, Ber. dtsch. chem. Ges. 73, 962 [1940].

28 J. B. Evans, J. Bacteriol. 55, 793 [1948].

\title{
Beziehungen zwischen Sulfonamiden und Folinsäure; durch Sulfonamide bedingtes Folinsäurebedürfnis von Bakterien
}

\author{
Von Ernst-Friedrich Möller, Friedrich Weygand und Adolf Wacker \\ Aus dem Kaiser-Wilhelm-Institut für medizinische Forschung, Institut für Chemie, Heidelberg, \\ und dem Chemischen Institut der Universität Heidelberg
}

(Z. Naturforschg. 4 b, 100-103 [1949]; eingegangen am 13. Mai 1949)

\begin{abstract}
In Übereinstimmung mit $\mathrm{A} \mathrm{u} \mathrm{h}$ a g e n lassen sich die Bakterien bezüglich der Hemmbarkeit durch Sulfonamide und der Aufhebbarkeit dieser Hemmung durch Folinsäure in 3 Gruppen einteilen. Wir fanden, daß alle bisher untersuchten Vertreter der Gruppe A gramnegativ, die der Gruppe $\mathrm{B}$ und $\mathrm{C}$ grampositiv sind. Es gelang, Bakterien der Gruppe B (folinsäuresynthetisierend) durch Passagen in sulfathiazol- und folinsäurehaltigem Medium folinsäurebedürftig zu machen.
\end{abstract}

$\mathrm{I}_{\mathrm{t}+\mathrm{s}}^{\mathrm{n}}$ n einer kürzlich erschienenen Mitteilung berichtete E. A uhagen ${ }^{1}$ über Beziehungen zwischen Folinsäurebedürfnis und Hemmbarkeit bei Bakterien. Er teilte die Bakterien in drei Gruppen ein:

Gruppe A wächst in folinsäurefreiem Nährmedium und ist durch Sulfonamide hemmbar. Die Hemmung durch Sulfonamide wird durch Folinsäure nicht aufgehoben.

1 E. A u h a g e n, Hoppe-Seyler's Z. physiol. Chem. 283, 195 [1948].
Grup p e B wächst ebenfalls in folinsäurefreiem Nährmedium und ist durch Sulfonamide hemmbar. Die Hemmung wird bereits durch Folinsäure in so kleinen Konzentrationen aufgehoben, wie sie zum Wachstum folinsäurebedürftiger Stämme ausreicht.

Gruppe C wächst nur in Anwesenheit von Folinsäure und ist durch Sulfonamide nicht hemmbar.

Wir haben uns seit längerer Zeit mit ähnlichen 
Fragen beschäftigt und sind an einem größeren Versuchsmaterial zu prinzipiell gleichen Ergebnissen gekommen. Zur Gruppe A gehören nach unseren Ergebnissen alle diejenigen Bakterien, bei denen keine Stämme vorhanden sind, die Folinsäure zum Wachstum benötigen ${ }^{2}$, und zwar: B. coli, B. proteus, B. fluorescens, B. typhi, B. paratyphi, B. dysenteriae und B. enteritidis. Zur Gruppe B und $\mathrm{C}$ gehören solche Bakterien, bei denen es folinsäurebedürftige und nicht-folinsäurebedürftige Stämme gibt ${ }^{2}$, und zwar: Staphylokokken, Streptokokken, Milchsäurebakterien und Enterokokken.

Auffällig ist, daß alle Bakterien der Gruppe A zu den gramnegativen, alle der Gruppe B und C zu den grampositiven Bakterien zählen. Es ist möglich, daß hier Beziehungen zwischen dem für die Gram-Färbung verantwortlichen Mg-Salz der Ribonucleinsäure ${ }^{3}$ und dem wahrscheinlichen Eingreifen von Folinsäure in die Synthese von Pyrimidinen und evtl. Purinen bestehen ${ }^{4}$. Stämme, die in folinsäurefreiem Nährmedium wachsen und durch Sulfonamide nicht hemmbar sind, haben

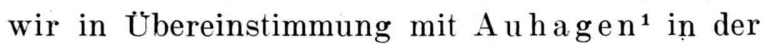
Natur ebenfalls bisher nicht gefunden.

Die Unterschiede der Gruppen A und B bei der Enthemmung von Sulfathiazol durch Folinsäure und mit ihr verwandte Verbindungen zeigen wir an zwei Vertretern, einem Coli- und einem Staphylokokken-Stamm (s. Tab. 1).

Es ergibt sich somit, daß bei E. coli nur $p$ Aminobenzoesäure und $p$-Aminobenzoyl-l-glutaminsäure, nicht aber Folinsäure und ihre.Abkömmlinge oder Vitamin $B_{12}$ Sulfathiazol vollständig enthemmen können. Dieser Befund steht im Einklang mit den Ergebnissen von J. O. L a mpen und M. J. Jones ${ }^{8}$, daß zwischen Folinsäure und Sulfonamiden bei B. coli kein Antagonismus besteht (s.a. R.Tschesche ${ }^{9}$ und E. A uhagen ${ }^{1}$ ). Der von L. J.Daniel und L.C. Norris ${ }^{10}$ aufgefundene kompetitive Antagonismus Sulfathiazol: Folinsäure tritt nur bei kleinen Konzentra-

2 E. F. Möller, F. Weygand u. A. Wa cker, Folinsäure als Wuchsstoff bei verschiedenen Bakterien; ein neuer Folinsäuretest mit Staph.aureus, Z. Naturforschg. 4 b, 92 [1949].

3 H. H e n r y u. M. S t a c e y, Brit. med. J. 1946, $950,4481$.

4 L. L. R o g e r s u. W. S h i ve, J. biol. Chemistry 172, 751 [1948].

5 Bei kleineren Sulfathiazolkonzentrationen $(\sim$ $16 \gamma / \mathrm{cm}^{3}$ ) bewirkt Folinsäure erst in hohen Konzentrationen eine Enthemmung.

\begin{tabular}{|c|c|c|}
\hline Substanz & $\begin{array}{c}\text { Gruppe A } \\
\text { B. coli } 1883 \mathrm{Co} \\
\%\end{array}$ & $\begin{array}{c}\text { Gruppe B } \\
\text { Staph.BüAAu } \\
\%\end{array}$ \\
\hline $\begin{array}{l}p \text {-Aminobenzoesäure . . } \\
p \text {-Aminobenzoyl. } l \text { - } \\
\text { glutaminsäure. . . . }\end{array}$ & $\begin{array}{l}+(100) \\
+(100)\end{array}$ & $\begin{array}{l}+(100) \\
+(100)\end{array}$ \\
\hline Pteroinsäure . . . . . & 0 & $+(100)$ \\
\hline Rhizopterin . . & 0 & $+(100)$ \\
\hline Folinsäure .. & $0^{5}$ & $+(90)$ \\
\hline Formyl-folinsäure . . . & 0 & $+(100)$ \\
\hline Teropterin ....... & 0 & $+(95)$ \\
\hline 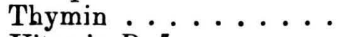 & $+(50-60)^{6}$ & $+(100)$ \\
\hline Vitamin $\mathrm{B}_{12}{ }^{7} \ldots \ldots$ & 0 & 0 \\
\hline
\end{tabular}

Tab. 1. Enthemmung von $133 \gamma / \mathrm{cm}^{3}$ Sulfathiazol. $+=$ Enthemmung; $0=$ keine Enthemmung.

tionen an Sulfathiazol und sehr hohen Konzentrationen an Folinsäure auf und dürfte auf eine geringfügige Freisetzung von $p$-Aminobenzoesäure aus Folinsäure zurückzuführen sein.

Außer $p$-Aminobenzoesäure wirkte in unseren Versuchen nur noch Thymin enthemmend, jedoch nicht vollständig, und zwar um so weniger vollständig, je höher die Sulfonamidkonzentration war. Versuche, die Enthemmung mit Thymin vollständig zu machen durch Zusatz von Substanzen, von denen z. Tl. bekannt ist, daß sie unter bestimmten Bedingungen Sulfonamide enthemmen, mißlangen. Untersucht wurden: die in der Nährlösung enthaltenen Purine, Pyrimidine, Aminosäuren und Vitamine in höheren Konzentrationen; weiterhin Tween 80, Redukton, Cholin, Inosit, Vitamin $B_{12}$ und Folinsäure, außerdem $p$-Aminobenzoesäure in Konzentrationen, die nur eine teilweise Enthemmung hervorrufen (keine additive Enthemmung durch $p$-Aminobenzoesäure + Thy$\min )$.

Bei dem nicht-folinsäurebedürftigen Staphylokokkenstamm enthemmen dagegen alle geprüften Verbindungen. Im Gegensatz zur $p$-Aminobenzoesäure, die bekanntlich in kompetitiver Weise Sulfonamide enthemmt, genügt bei Folinsäure bereits eine sehr kleine, praktisch gleichbleibende

6 E. L. R. Stokstad fand, daß Thymin in Gegenwart einer Purinbase bei L. casei nur $50 \%$ des mit Folinsäure maximal erzielbaren Wachstums ergab.

7 Für die Überlassung von Vitamin $B_{12}$ danken wir Hrn. Dr. R. T. M a jor von M e r c k \& Co., Rahway, USA, bestens.

${ }^{8}$ J. O. L a m p en u. M. J. J o n es, J. biol. Chemistry 164, 485 [1946].

9 R. T s c h e s c h e, Z. Naturforschg. 2 b, 10 [1947]; R. Tschesche, K. Soehring u. K. H a r d e r, Z. Naturforschg. 2 b, 244 [1947].

10 L. J. D a n i e l u. L. C. N o r r is, J. biol. Chemistry 170, 747 [1947]. 
Menge $\left(\sim 0,005 \gamma / \mathrm{cm}^{3}\right)$, um Sulfathiazol über ein sehr grỏßes Konzentrationsgebiet $\left(0,5-500 \gamma / \mathrm{cm}^{3}\right)$ zu enthemmen. Dieser nicht-kompetitive Antagonismus steht in Übereinstimmung mit den Befunden von Lampen und Jonesi1; er wurde dahingehend ausgelegt, daß die Sulfonamide die Synthese der Folinsäure, nicht aber ihre Funktion hemmen.

Ferner wirken die formylierten Verbindungen (Rhizopterin und Formyl-folinsäure) in kleineren Konzentrationen als die nicht-formylierten. Redukton, das nach H.S. Forrest ${ }^{12}$ als Zwischenprodukt der biologischen Folinsäuresynthese in Bakterien vermutet wird, enthemmte nicht.

Es war nun interessant, zu sehen, ob Bakterien der sulfonamidempfindlichen, nicht-folinsäure bedürftigen Gruppe B nach Sulfonamidfestigung in Stämme übergehen, die sich wie die der Gruppe C verhalten, die von Natur aus sulfonamidresistent sind und zum Wachstum Folinsäure benötigen.

Die Prüfung dieser Fragestellung war naheliegend, weil in der Natur unter den gleichen Bakterienarten Stämme vorkommen, die entweder zur Gruppe B oder zur Gruppe C gehören.

Die Versuche wurden in dem halbsynthetischen Folinsäuretest-Medium durchgeführt, das wir in unseren früheren Arbeiten benutzt haben ${ }^{2}, p$ Aminobenzoesäure wurde weggelassen. So wurde der nicht-folinsäurebedürftige Staphylokokkenstamm BüAAu in folinsäure- und sulfathiazolfreiem Medium vorgezüchtet, dann wurde er in lau-. fenden Passagen in demselben Medium in Gegenwart von $500 \gamma / \mathrm{cm}^{3}$ Sulfathiazol und $0,001 \mathrm{\gamma} / \mathrm{cm}^{3}$ Folinsäure weitergezüchtet. Der Stamm wuchs zunächst schlecht, dann von Passage zu Passage. stärker. Nach 2-3 Passagen war das normale Wachstum wieder ereicht, oder sogar noch etwas überschritten (s. Abb.1), Es wurde nun nach jeder Passage (24 Stdn.) die Hemmbarkeit durch Sulfathiazol (Abb. 2a) und das Folinsäurebedürfnis (Abb. 2 b) geprüft.

Es ergab sich, daß eine rasche, kontinuierlich sich steigernde Folinsäurebedürftigkeit auftritt, die nach etwa 3-4 Passagen vollständig erreicht ist. Diese Folinsäurebedürftigkeit geht rasch wieder verloren, denn überimpft man nach 2 Passagen in sulfathiazolfreiem, folinsäurehaltigem Medium

11 J. O. L a mp e n u. M. J. J o n e s, J. biol. Chemistry 164, 485 [1946]; 166, 435 [1946].

${ }_{12}$ H. S. F or rest u. J. W a l ker, Nature [London] 161, 721 [1948]. einmal in folinsäurefreies, das andere Mal in folinsäurehaltiges Medium, so beobachtet man gleich gutes Wachstum; das Verhalten des Ausgangsstammes ist wiederhergestellt. Man erreicht dies in einer Passage, wenn $p$-Aminobenzoesäure an Stelle von Folinsäure verwendet wird.

Man erhält auch Stämme mit Folinsäurebedürftigkeit, wenn man die Passagen in Gegenwart von Sulfathiazol + Thymin durchführt, desgleichen nur in einem sulfathiazolhaltigen Medium, wobei im letzteren Falle die Stämme fast kein sichtbares Wachstum zeigen. Werden aber die Passagen in Sulfathiazol $+p$-Aminobenzoesäure durch-

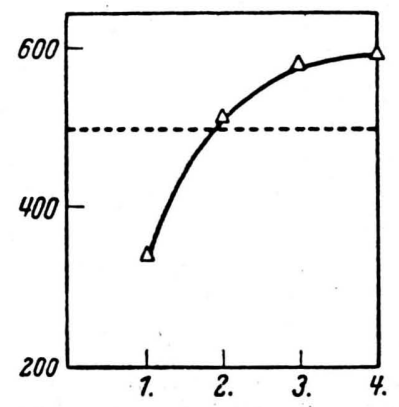

Abb. 1. Wachstum von Staph. BüAAu in laufenden Passagen in einem Medium mit $500 \gamma / \mathrm{cm}^{3}$ Sulfathiazol $+0,001 \mathrm{\gamma} / \mathrm{cm}^{3}$ Folinsäure nach je 24 Stdn. Ordinate: Trübungswerte; Ablesung an der Extinktionsskala des L a n g e - Photometers $\times 1000$. Abszisse: Nr. der Passage. ..... normales Wachstum.

geführt, so ändert sich die Eigenschaft des Stammes nicht!

In einem unterscheiden sich die Eigenschaften des künstlich folinsäurebedürftig gemachten Stammes von denen der Gruppe C: Bei ihm kann Folinsäure nicht nur durch ihre Abkömmlinge und Thymin, sondern auch durch $p$-Aminobenzoesäure ersetzt werden. Fünf weitere Staphylokokkenstämme zeigten gleiches Verhalten.

Versuche, Vertreter der Gruppe A (zwei Stämme von B.coli) nach den oben geschilderten Methoden in folinsäurebedürftige Stämme umzuzüchten, waren erfolglos.

Die Umwandlung von Bakterien der Gruppe B in folinsäurebedürftige und das leichte Zurückschlagen in die Ausgangsstämme kann folgendermaßen gedeutet werden. Bei den Passagen in Sulfạthiazol + folinsäurehaltigem Medium wird die an Eiweiß gebundene $p$-Aminobenzoesäure nach dem Massenwirkungsgesetz durch Sulfathiazol ausgetauscht. Dadurch wird die Synthese der 
Folinsäure aus $p$-Aminobenzoesäure zwar blokkiert, aber das Bakterium kann wachsen, weil Folinsäure im Nährmedium vorhanden ist. Nach mehreren Passagen sind die Haftstellen der $p$ Aminobenzoesäure offenbar alle durch Sulfathiazol besetzt. Dadurch kann die Folinsäuresynthese nicht in Gang kommen. Erst durch das Wachsen in folinsäurehaltigem, sulfathiazolfreiem Medium wird nach und nach, offenbar in etwa der gleichen Anzahl von Passagen, wie zur Erlangung der Folinsäurebedürftigkeit notwendig waren, die Sulfathiazolkonzentration in den Bakterien so teres ein, daß $p$-Aminobenzoesäure die Folinsäure als Wuchsstoff bei den folinsäurebedürftig gemachten Stämmen der Gruppe B ersetzen kann, da sie das in den Bakterien gebundene Sulfathiazol sofort verdrängt und dadurch die Folinsäuresynthese wieder ermöglicht. Damit erklärt sich auch der Verlust der Folinsäurebedürftigkeit bereits nach einer Passage in einem Medium, das $p$-Aminobenzoesäure enthält.

Nach den hier mitgeteilten Befunden ist es möglich, daß viele der in der Literatur beschriebenen sulfonamidresistent gemachten Stämme solche
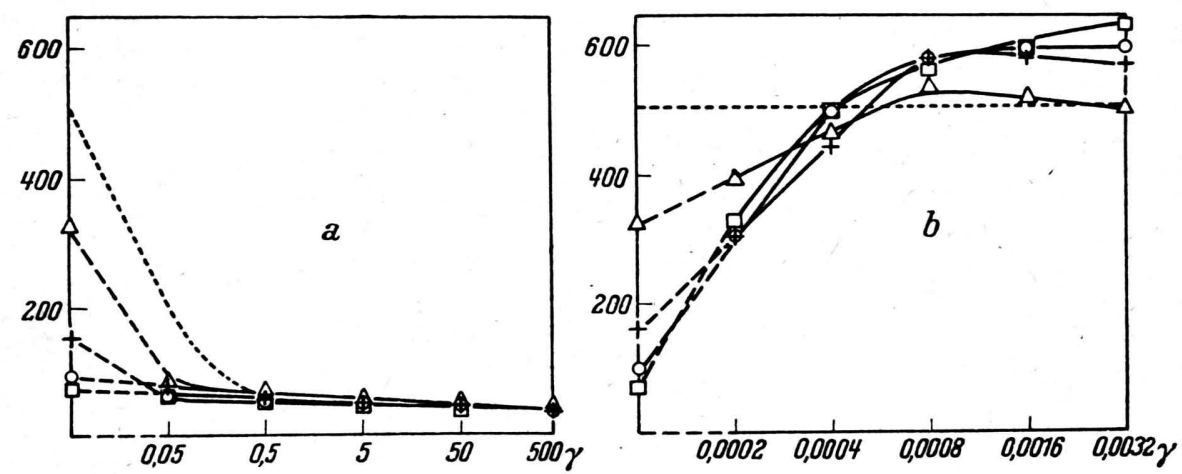

Abb. 2. Vergleich von Sulfathiazolhemmbarkeit und Folinsäurebedürfnis von Staph. BüAAu während laufender Passagen in sulfathiazol- und folinsäurehaltigem Medium nach 14 Stdn. Ordinaten: wie Abb. 1. Abszissen: Abb. 2 a Mengen an Sulfathiazol in $\gamma / \mathrm{cm}^{3}$, Abb. 2 b Mengen an Folinsäure in $\gamma / \mathrm{cm}^{3}$. - . . beimpft aus Medium ohne Sulfathiazol und ohne Folinsäure (Ausgangsstamm); beimpft aus Medium mit $500 \gamma / \mathrm{cm}^{3}$ Sulfathiazol und $0,001 \mathrm{\gamma} / \mathrm{cm}^{3}$ Folinsäure $\triangle \longrightarrow \triangle 1$. Passage, $+\longrightarrow+$ 2. Passage, $0 \longrightarrow 0$ 3. Passage, $\square-\square$. Passage.

weit herabgesetzt, daß die Eigensynthese der Folinsäure und damit das Wachstum in folinsäurefreiem Medium wieder möglich ist.

Ersetzt man bei der Züchtung folinsäurebedürftiger Stämme Folinsäure durch Thymin, so ändert sich an dem Mechanismus nichts, da das Thymin als das Endprodukt einer durch Folinsäure katalysierten Reaktion angesehen wird.

Im Einklang mit den oben geschilderten Vorstellungen steht, daß in Anwesenheit von Sulfathiazol $+p$-Aminobenzoesäure gezüchtete Stämme der Gruppe B kein Folinsäurebedürfnis erlangen: Die Folinsäuresynthese kann ungehindert verlaufen, weil die anwesende $p$-Aminobenzoesäure die die Folinsäuresynthese katalysierenden Stellen von Sulfathiazol frei hält. In den vorgeschlagenen Mechanismus ordnet sich auch ohne wei- sind, die die Eigenschaft der Sulfonamidresistenz nur vorübergehend angenommen haben in dem Sinne wie unsere umgewandelten Stämme. Dafür spricht, daß die vielfach verwendeten natürlichen Medien nach Stokes, Guness und Foster ${ }^{13}$ rélativ viel Folinsäure, aber wenig $p$-Aminobenzoesäure enthalten. Fügt man jedoch einem natürlichen Nährmedium $p$-Aminobenzoesäure hinzu, so werden z. B. Pneumokokken ${ }^{14}$, E. coli und Staphylokokken ${ }^{15}$ nicht-resistent gegen Sulfonamid.

13 J. L. Stokes, M. Guness u. J. W. Foster, J. Bacteriology 47, 893 [1944].

${ }^{14} \mathrm{H}$. B loch u. P. Bernoulli, Schweiz. med. Wschr. 74, 1341 [1944]; Helv. chim. Acta 27, 362 [1944].

${ }^{15}$ E. S traus S, H. J. D ing le u. M. Finland, J. Immunology 42, 313, 331 [1941]. 